\title{
Effects of Sevoflurane and Adenosine Receptor Antagonist on the Sugammadex-Induced Recovery from Rocuronium-Induced Neuromuscular Blockade: An Ex-vivo Study
}

\author{
Yong Beom Kim ( $\nabla$ fred0314@gilhospital.com ) \\ Gachon University Gil Medical Center https://orcid.org/0000-0003-2369-6525 \\ Jae-Moon Choi \\ Asan Medical Center, Ulsan University, College of Medicine \\ Chungon Park \\ Gachon University Gil Medical Center \\ Hey-Ran Choi \\ Inje University Seoul Paik Hospital \\ Junyong In \\ Dongguk University Ilsan Hospital \\ Hong-Seuk Yang \\ Daejeon Sun Hospital
}

Research article

Keywords: Acetylcholine, Neuromuscular blockade, Neuromuscular blocking agent, Rocuronium, Sevoflurane, Sugammadex

Posted Date: August 7th, 2020

DOI: https://doi.org/10.21203/rs.3.rs-43525/v1

License: (c) (i) This work is licensed under a Creative Commons Attribution 4.0 International License. Read Full License 


\section{Abstract}

Background: Sevoflurane affects on the A1 receptor in the central nervous system (CNS) and potentiates the action of neuromuscular blocking agents. In the present study, we investigated whether sevoflurane (SEVO) has the ability to potentiate the neuromuscular blocking effect of rocuronium and if the specific antagonist of adenosine receptor (SLV320) can reverse this effect.

Methods: Phrenic nerve-hemidiaphragm tissue specimens were obtained from forty Sprague-Dawley (SD) rats. The specimens were immersed in an organ bath filled with Krebs buffer and stimulated by a train-of-four (TOF) pattern using indirect supramaximal stimulation at $20 \mathrm{~s}$ intervals. The specimens were randomly allocated to control, 2-chloroadenosine (CADO), SEVO, or SLV320+SEVO groups. In the CADO and SLV320+SEVO groups, CADO and SLV320 were added to the organ bath from the start to a concentration of $10 \mu \mathrm{M}$ and $10 \mathrm{nM}$, respectively. We then proceeded with rocuronium-induced blockade of $>95 \%$ depression of the first twitch tension of TOF (T1) and TOF ratio (TOFR). In the SEVO and SLV320+SEVO groups, SEVO was added to the Krebs buffer solution to concentration of $400-500 \mu \mathrm{M}$ for 10 min. Sugammadex-induced T1 and TOFR recovery was monitored for $30 \mathrm{~min}$ until $>95 \%$ of $\mathrm{T} 1$ and $>0.9$ of TOFR were confirmed, and the recovery pattern was compared by plotting these data.

Results: There were no significant differences in the recovery pattern between the control and SEVO groups. However, there were significant differences between the SEVO and SLV320+SEVO groups.

Conclusion: Sevoflurane potentiates of rocuronium-induced neuromuscular blocking effect and delays sugammadex-induced recovery from neuromuscular blockade.

\section{Background}

Sevoflurane is a commonly used inhalation anaesthetics in clinical settings. Although the effects of inhalation anaesthetics cannot be explained by a single molecular mechanism, it is known that sevoflurane has a tendency to activate adenosine A1 receptor [1]. The hypnotic or soporific effects of adenosine have been described in animals [2] and humans [3]. Furthermore, specific antagonists of the adenosine receptor, such as theophylline, can decrease the sedation effect of propofol [4] and sevoflurane [5]. Such effects are due to the blockade of effects of sevoflurane or propofol on adenosine receptors. At the neuromuscular junction, adenosine receptors, together with presynaptic muscarinic acetylcholine receptors (mAChRs), modulate acetylcholine (ACh) release upon neuronal firing and regulate each other as a facilitatory or inhibitory receptor [6-8]. When these receptors are modulated and their functions are affected by specific agonists and antagonists, spontaneous and evoked release of ACh at the neuromuscular junction has been observed. These changes, in turn, affect the responses of muscle tension to variable neuronal stimulation [9].

Sugammadex, a y-cyclodextrin derivative, can reverse the effect of aminosteroidal neuromuscular blocking agents by encapsulating and inactivating them [10-12]. This effect does not influence the amount of ACh at the neuromuscular synaptic junction and this is different from the mechanism of 
classic reversal strategy to increase ACh using cholinesterase inhibitors such as neostigmine [13]. We hypothesized that, during sugammadex-induced recovery from neuromuscular blockade, the muscle tension might be influenced by modulation of the presynaptic release of ACh. We also hypothesized that the modulation of ACh release might be responsible for the potentiation of the neuromuscular blockade effect of sevoflurane. To confirm these hypotheses, here, we performed ex-vivo experiments using rodent phrenic nerve/hemidiaphragm tissue specimens. We investigated the time course of sugammadexinduced recovery from rocuronium-induced neuromuscular blockade in tissue specimens in an organ bath with adenosine $A 1$ receptor agonist, sevoflurane, or sevoflurane and adenosine $A_{1}$ receptor specific blocker.

\section{Methods}

\section{Basic study design and sample preparation}

The study protocol was approved by the Ethics Committee of the Laboratory of Animal Research, Asan Institute of Life Science (Seoul, Republic of Korea) on September 1, 2018 (Protocol No.2018-13-198). Forty male Sprague-Dawley (SD) rats with average weight of $245.45 \mathrm{~g}$ (range 225.0-296.9 g) were used in the study. All rats were bred at a constant temperature of $22^{\circ} \mathrm{C}$ under a regular diurnal cycle. They were provided food and water ad libitum. We performed surgical procedures for harvesting tissue specimen after confirming that the rats didn't respond by pinching their hindlimb. Additional sacrifice methods for SD rats were not necessary in this study because rats were expired as soon as the thoracic cages were harvested during the preparation. Inclusion criteria was that the initial T1 twitch tension showed more than $100 \mathrm{mN}$. Data were excluded when the T1 twitch tensions were gradually decreased before adding study drugs or when the maximum recovery of $\mathrm{T} 1$ twitch tension was less than $50 \mathrm{mM}$. Urethane, 2chloroadenosine (CADO), and SLV320 was purchased from Sigma-Aldrich Korea Ltd, (Gyeonggido, Republic of Korea). CADO stock solution was stored at $-20^{\circ} \mathrm{C}$ in a refrigerator and thawed before used. Stock solutions were discarded 2 weeks after preparation if unused.

\section{Study protocol}

Each rat was anaesthetized with intraperitoneal injection of urethane $(500 \mu \mathrm{g} / \mathrm{kg})$. The thoracic cage was immediately isolated, and the phrenic nerve/hemidiaphragm tissue was obtained. The sampled tissues were immersed in Krebs buffer solution $\left(120 \mathrm{mM} \mathrm{NaCl}, 2.5 \mathrm{mM} \mathrm{CaCl}_{2}, 4.7 \mathrm{mM} \mathrm{KCl}, 1.2 \mathrm{mM} \mathrm{MgSO}_{4}\right.$, $1.2 \mathrm{mM} \mathrm{KH} 2 \mathrm{PO} 4,25 \mathrm{mM} \mathrm{NaHCO}_{3}$, and $11 \mathrm{mM}$ a-D-glucose) maintained at $35^{\circ} \mathrm{C}$ and $95 \% \mathrm{O}_{2}$ and $5 \% \mathrm{CO}_{2}$ with continuous bubbling to ensure tissue viability throughout the experimental session. Sizes and weights of each tissue were measured and compared between groups (Table 1). The tissues were fixed to a frame with electrodes and then immersed in a $100 \mathrm{ml}$ organ bath containing $75 \mathrm{ml}$ of oxygenated Krebs buffer solution. Subsequently, $40 \mathrm{mN}$ resting tension was applied to the tendinous portion of the diaphragm of each sample hooked to a Grass FT03 Force Transducer (Grass Technologies, West Warwick, RI, USA). The phrenic nerve was fixed to a platinum bipolar electrode and stimulated using a Grass S88 Stimulator (Grass Technologies). Supramaximal stimulation with a square wave pulse of 
$0.2 \mathrm{~ms}$ was administered every $20 \mathrm{~s}$ at trains of $2 \mathrm{~Hz}$ for $2 \mathrm{~s}$ (train of four (TOF) stimulation). All waveforms were acquired and stored using the PowerLab 4/26 Data Acquisition System (AD Instruments, Sydney, Australia) and LabChart 7 Software (AD Instruments, Colorado Springs, CO, USA), respectively.

Table 1

Characteristics of Sprague-Dawley rats and tissue specimen

\begin{tabular}{|c|c|c|c|c|}
\hline & $\begin{array}{l}\text { Control } \\
(n=10)\end{array}$ & $\begin{array}{l}\text { SEVO } \\
(n=10)\end{array}$ & $\begin{array}{l}\text { SEVO + SLV320 } \\
(n=10)\end{array}$ & $\begin{array}{l}\text { CADO } \\
(n=10)\end{array}$ \\
\hline BW (g) & $246.36 \pm 13.78$ & $239.38 \pm 5.94$ & $255.70 \pm 16.26$ & $240.48 \pm 6.23$ \\
\hline wWt (mg) & $123.91 \pm 18.53$ & $123.08 \pm 18.34$ & $121.00 \pm 13.70$ & $122.78 \pm 18.64$ \\
\hline Size $\left(\mathrm{mm}^{2}\right)$ & $133.04 \pm 28.94$ & $125.38 \pm 24.63$ & $127.20 \pm 33.93$ & $128.51 \pm 23.52$ \\
\hline \multicolumn{5}{|c|}{ Data are expressed as mean $\pm S D$. There were no significant differences among the groups $(P>0.05$} \\
\hline
\end{tabular}

The phrenic nerve/hemidiaphragm tissues were randomly allocated to the control, CADO (CADO $10 \mu \mathrm{M})$, SEVO (sevoflurane 400-500 $\mu \mathrm{M}$ ), or SLV320 group (sevoflurane 400-500 $\mu \mathrm{M}+$ SLV320 $10 \mathrm{nM}$ ) after generating random number by using following equation in Microsoft Excell 2010 program (Microsoft Office ${ }^{\circledR}$, Microsoft Corporation, USA); $f_{\mathrm{x}}=\left(\operatorname{INT}\left(\operatorname{RAND}()^{* 4}\right)\right)+1$. Group blinding was not available because SEVO is volatile and its scent is pungent. Twitch tensions were serially monitored during a $30 \mathrm{~min}-$ stabilization time. Thereafter, Krebs buffer solution, SLV320, or CADO stock solution, of same volume, was added to the organ bath. A $500 \mu \mathrm{g}$ loading dose of rocuronium (Esmeron®; MSD Korea, Seoul, Republic of Korea) and $250 \mu \mathrm{g}$ boost dose of rocuronium were subsequently added into the organ bath. The subsequent boost dose was added 10 min after the previous dose or when five consecutive T1 twitch tension depressions were less than $3 \%$ of the previous $\mathrm{T} 1$ twitch tension. The boost doses were stopped when $95 \%$ or more depression of the T1 was achieved. The loading dose was set as the dose at which the T1 twitch tension did not change after adding the loading dose and the TOF ratio (TOFR) changes were within $3 \%$ of those achieved before adding the loading dose. The boost dose was set as the first dose at which the $\mathrm{T} 1$ twitch tension changed and the total boost doses were within 10 . After confirming that the T1 twitch tensions disappeared, sevoflurane was added to the SEVO and SLV320 groups for 10 min to a concentration of $400-500 \mu \mathrm{M}$, wherease the other groups were allowed to rest for $10 \mathrm{~min}$. After $10 \mathrm{~min}$ of reaction, equimolar doses of sugammadex, which were to react 1:1 to the rocuronium used to create > 95\% depression of 11 twitch tension, were added to each group to recover from rocuronium-induced neuromuscular blockade. Recovery patterns were monitored and plotted for 30 min or until the T1 twitch tension recovery was $>95 \%$ and TOFR was $\geq 0.9$. The study protocol is summarized in Fig. 1 .

\section{Statistical analysis}


The first aim of this study was to compare the time required in each group to attain $>95 \%$ recovery in T1. To achieve this goal, the T1 twitch tensions were serially monitored and recorded; these responses were plotted as regression curves and compared among the groups. T1 recovery and TOFR recovery were achieved simultaneously until TOFR was $>0.9$. The second aim was to compare the variables obtained during sugammadex-induced recovery from neuromuscular blockade. In a clinical practice, the recovery index ( $\mathrm{Rl}$, the interval of $\mathrm{T} 1$ recovery from $25-75 \%)$ is a good marker for comparing the recovery rates from neuromuscular blockade. We performed inter-group comparisons of the time interval of T1 twitch tension recovery at $10 \%, 25 \%, 50 \%, 75 \%$, and $95 \%$.

There are many issues with performing sample size estimation in ex-vivo neuromuscular studies. Previous studies have suggested that about 10 animals per group are needed to achieve statistically significant results $[14,15]$. In the present study, the sample size was calculated based on the previous experiment and pilot study, which suggested that 10 samples per group were sufficient at $a=0.05$, power $=0.80$, and a dropout rate of $10 \%$.

Results are expressed as mean \pm standard deviation (SD). All doses were converted and expressed in $\mu \mathrm{mol} / \mathrm{L}(\mu \mathrm{M})$. Time is expressed in min. Graphs were plotted, and statistical analyses were carried out

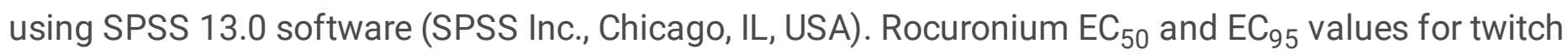
tension were calculated by fitting nonlinear regression curves to group data. We used the following equation for T1 depression; $y=50+50 \sin (\Omega x+b)$, where y represents the T1 tension, $x$ represents the recovery time, b represents half of the time required to achieve $T 1>95 \%$, and $\Omega$ represents the slope of the regression curve $\left(R^{2}=0.87\right)$. Start points were set as the time of administration of sugammadex and the time when T1 twitch tension recovered to $10 \%$, and the slopes are represented as $\Omega_{1}$ and $\Omega_{2}$, respectively. We considered the RI as the time interval from $25-75 \%$ of $\mathrm{T} 1 \mathrm{twitch}$ tension recovery, and compared the RI among the groups. We also used the following equation for TOFR; $y=1-\lambda x^{2}, w h e r e y$ and $x$ represent TOFR and recovery time, respectively, while $\lambda$ represents the slope of the regression curve $\left(R^{2}=0.83\right)$. Differences in continuous variables among the groups were analysed using analysis of variance, followed by the Bonferroni method for multiple pairwise comparisons. The mean group values of $\Omega$ s and $\lambda$ were compared using the Mann Whitney $U$ test. Statistical significance was set at $p<0.05$.

\section{Results}

There were no differences in the average body weight of rats, wet weight of tissue specimens, and dose of rocuronium used among the groups (Table 1). We plotted the recovery data of T1 and TOFR of each group and compared their slopes (represented as $\Omega$ and $\lambda$, respectively). When T1 twitch tension recovery was plotted with the start point as the time of sugammadex administration, there were no significant differences on $\Omega_{1}$ between groups (Fig. 2A, B). The data of the CADO group were not included in this comparison because most of the T1 twitch tensions in the CADO group were reappeared 5 min after the administration of sugammadex and did not recover within $30 \mathrm{~min}$. When the start point was set as the time when the T1 twitch tension recovered to $10 \%$, there were significant differences in $\Omega_{2}$ of the CADO 
and SEVO groups compared with that of control and SLV320 + SEVO groups (Fig. 2C, D). We also compared the recovery time interval from $25-75 \%$ recovery of $\mathrm{T} 1$ (RI) among the groups. The RIs of the CADO and SEVO groups were significantly different from those of the other groups, although there were no significant differences between the control and SEVO + SLV320 group (Table 2, $\mathrm{P}<0.05$ ). A comparison of TOFR recovery revealed no significant differences among the groups with the equation that we adopted, but the TOFRs of control and SEVO + SLV320 groups were well above 0.7 from the start, showed temporary decrease to about 0.5 , and then recovered again. In the CADO and SEVO groups, although some patterns of TOFR recovery progression were similar to those of the control and SEVO + SLV320 groups, some patterns showed a linear progression (Fig. 3).

Table 2

Comparison of recovery progression and recovery index.

\begin{tabular}{|c|c|c|c|c|}
\hline & $\begin{array}{l}\text { Control } \\
(n=10)\end{array}$ & $\begin{array}{l}\text { SEVO } \\
(n=10)\end{array}$ & $\begin{array}{l}\text { SEVO + SLV320 } \\
(n=10)\end{array}$ & $\begin{array}{l}\text { CADO } \\
(n=10)\end{array}$ \\
\hline$\Omega_{1}$ & $0.975 \pm 0.237$ & $1.027 \pm 0.104$ & $0.954 \pm 0.234$ & - \\
\hline$\Omega_{2}$ & $0.877 \pm 0.202$ & $1.002 \pm 0.092^{\star}$ & $0.898 \pm 0.202$ & $0.999 \pm 0.056^{\star}$ \\
\hline RI (min) & $6.034 \pm 1.791$ & $8.531 \pm 2.113^{\dagger}$ & $5.534 \pm 1.852$ & $14.949 \pm 4.498^{\dagger}$ \\
\hline \multicolumn{5}{|c|}{$\begin{array}{l}\text { Data are expressed as mean } \pm S D . *: P<0.050 \text { compared with the control or SEVO }+ \text { SLV320 group, } t: \\
P<0.050 \text { compared with the control or SEVO }+S L V 320 \text { groups. }\end{array}$} \\
\hline \multicolumn{5}{|c|}{$\begin{array}{l}\text { Abbreviations: CADO, 2-chloroadenosine group; SEVO, sevoflurane; } \Omega_{1} \text {, regression slope when the zero } \\
\text { point was set as the time of sugammadex administration; } \Omega_{2} \text {, regression slope when the zero point } \\
\text { was set as the time of } 10 \% \mathrm{~T} 1 \text { twitch tension recovery. }\end{array}$} \\
\hline
\end{tabular}

\section{Discussion}

In the present study, we demonstrated that sevoflurane affects the $A_{1}$ receptor at the neuromuscular junction and delays sugammadex-induced recovery from rocuronium-induced neuromuscular blockade. Adenosine acts as an $A_{1}$ agonist at a low concentration (300 nM), and as an $A_{2 A}$ agonist at high concentration $(>1 \mu \mathrm{M})[16]$. Furthermore, it has been demonstrated that enflurane and sevoflurane have the ability to activate adenosine $A_{1}$ receptors in an in-vitro culture of rat hippocampus [1]. Aminophylline is a nonselective antagonist of the adenosine receptor [17], and it can decrease the sedation effects of sevoflurane [5, 18], but not the anaesthesia induced by desflurane [4]. Sevoflurane is one of the most potent volatile anaesthetics that potentiates the effect of neuromuscular blocking agents [19]. The present study results are in consistent with these findings. Sugammadex has no effect on the neuromuscular junction and ACh release. Sugammadex-induced recovery from rocuronium-induced neuromuscular blockade is dependent on the relative concentration of rocuronium and ACh at the neuromuscular junction. Thus, the T1 twitch response to the indirect nerve stimulation reappears in the presence of a large amount of ACh molecules at the neuromuscular junction, because the ACh molecules 
have a greater chance to attach to AChR than rocuronium. When the action of the receptor or channel related to the release of ACh was modulated and the release of ACh in the neuromuscular presynaptic membrane was reduced, there was a delay in recovery from neuromuscular blockade [14]. In the present study, the modulation of adenosine receptor with CADO and sevoflurane, delayed T1 recovery and resulted in a low recovery index compared with those of the control (no receptor modulation). We speculated that the modulation of the $A_{1}$ receptor at the presynaptic membrane of the neuromuscular junction by sevoflurane could partially induce delayed recovery from neuromuscular blockade. We did not change the current for phrenic nerve stimulation and thus, the magnitude of indirect stimulation to the phrenic nerve might not have changed. However, in this study, the amount of ACh released per indirect stimulation might have reduced during sugammadex-induced recovery from neuromuscular blockade because of the activation of the A1 receptor in the neuromuscular junctions by sevoflurane. Furthermore, sugammadex binds to rocuronium only outside the neuromuscular junction, and there is a concentration difference in rocuronium between the neuromuscular junction and organ bath; consequently, rocuronium is removed from the neuromuscular junction. In the present study, the decrease in rocuronium concentration at the neuromuscular junction was thought to be similar among all groups because we used equimolar dose of sugammadex and rocuronium in the organ bath. Rocuronium molecules were able to bind to the AChRs at the postsynaptic membrane because the amount of ACh released was less at the same stimuli that caused presynaptic A1 receptor modulation by sevoflurane. This might have resulted in the delayed recovery from rocuronium-induced neuromuscular blockade in the present study.

The present study had some limitations. First, this was an ex-vivo study. We extracted phrenic nervehemidiaphragm tissue specimens after sacrificing SD rats. During this phase, although we handled the specimen in the Krebs buffer solution with $95 \% \mathrm{O}_{2} / \mathrm{CO}_{2}$ gas bubbling, transient hypoxemia and tissue damage were inevitable. Furthermore, pharmacokinetic component was abolished during all phases of the experiment. To compensate for these limitations, we cautiously performed the following steps. (1) tissue specimens were extracted and immersed in a Petri dish and organ bath containing oxygenated Krebs buffer solutions throughout the experiment to minimize tissue hypoxia. (2) Maintaining the concentration of sevoflurane in the organ bath was another challenge. In the clinical setting, sevoflurane is supplied using an exclusive vaporizer and a closed circuit system, and its concentration is expressed as vol\%. However, in the present study, it was difficult to develop a closed circuit system because frames, electrods, and strings connected to the force transducer were out from the orifice of organ bath. Furthermore, scavenging system of our laboratory was not suitable for use with volatile anesthetics. Instead, to minimize air pollution in the laboratory, we had to find alternative method simulating a closed circuit sustem to apply sevoflurane. We sealed the organ bath with a flexible film and added sevoflurane intermittently to achieve the desired concentration in the Krebs buffer solution. In a clinical experiment, it took about $40 \mathrm{~min}$ for sevoflurane to equilibrate between blood and muscle component and make effect on muscle $[20,21]$. However, in some studies, sevoflurane was only needed 10 min for sevoflurane to make effect [21]. In our experiment, we shortened this reaction time to $10 \mathrm{~min}$ by applying sevoflurane directly to the environment of tissue specimen. This was quite different approach comparing with the clinical setting. Moreover, as sevoflurane is volatile, it is very difficult to maintain the designated 
concentration of sevoflurane in the organ bath, and fluctuation in its concentration was inevitable. The dose of sevoflurane was selected as $400-500 \mu \mathrm{M}$ at the point of initiation of recovery. This was determined before the main experiment by performing a pilot study and by referring to a previous study [22]. Second, this was a functional study, not an immunochemistry study. We performed this experiment by measuring the tension generated by diaphragm contraction. That is, we deduced the results in an indirect manner, as the tension is thought to be driven by the ACh concentration differences at the neuromuscular junction. For accurate data, timely measurement of the amount of ACh released during serial indirect stimulation is required. However, we could not find ideal method for this. The ACh concentrations should be measured repeatedly at $20 \mathrm{~s}$ interval. We performed a conventional functional study that is commonly used in neuromuscular studies. To obtain more convincing results, a more suitable immunohistochemical study should be conducted in the future.

\section{Conclusion}

sevoflurane potentiates the effects of neuromuscular blocking agent partially by modulating the adenosine $A_{1}$ receptor at the neuromuscular junction. Furthermore, it affects sugammadex-induced neuromuscular recovery after rocuronium-induced neuromuscular blockade. Although the results were obtained in an ex-vivo environment, they suggest that if sevoflurane is used as the main anaesthetic drug, recovery from neuromuscular blockade can be delayed at the end of surgery and, unlike it is expected, that sugammadex-induced recovery from neuromuscular blockade also be delayed.

\section{Abbreviations}

Acetylcholine (ACh), acetylcholine receptor (AChR)CADO, 2-chloroadenosine; SEVO, sevoflurane; SD, Sprague-Dawley rats; TOF, train-of four stimulation. muscarinic acetylcholine receptors (mAChRs).

\section{Declarations}

\section{Ethics approval and consent to participate}

The study protocol was approved by the Ethics Committee of the Laboratory of Animal Research, Asan Institute of Life Science (Seoul, Republic of Korea) on September 1, 2018 (Protocol No.2018-13-198).

\section{Consent for publications}

N/A

\section{Availability of data and material}


Raw data in this study will not be shared. If there are anyone who need the raw data of this study, please contact the corresponding author (fred0314@gilhospital.com).

\section{Competing interests}

The authors have no potential conflicts of interest to declare in association with this work.

\section{Funding}

There are no fundings in the present study.

\section{Authors contributions}

YBK and HSY designed the study, wrote the manuscript, YBK, JC, HC and HSY analyzed and interpreted the data. YBK JC, HC, JI and CP collected and statistical analyses the data. YBK wrote manuscript and all authors read and revised manuscript body. JC and HSY performed political and external relation work.

\section{References}

1. Tas PW, Eisemann C, Roewer N. Indirect activation of adenosine A1 receptors in cultured rat hippocampal neurons by volatile anaesthetics. Eur J Anaesthesiol 2005; 22: 694-702.

2. Haulilca I, Ababei L, Branisteanu D, Topoliceanu F. Preliminary data on the possible hypnogenic role of adenosine. J Neurochem 1973; 21: 1019-20.

3. Segerdahl M, Ekblom A, Sandelin K, Wickman M, Sollevi A. Peroperative adenosine infusion reduces the requirements for isoflurane and postoperative analgesics. Anesth Analg 1995; 80: 1145-9.

4. Turan A, Kasuya Y, Govinda R, Obal D, Rauch S, Dalton JE, et al. The Effect of Aminophylline on Loss of Consciousness, Bispectral Index, Propofol Requirement, and Minimum Alveolar Concentration of Desflurane in Volunteers. Anesth Analg 2010; 110: 449-54.

5. Turan A, Memiş D, Karamanlioğlu B, Çolak A, Pamukçu Z, Turan N. Effect of aminophylline on recovery from sevoflurane anaesthesia. Eur J Anaesth 2002; 19: 452-4.

6. Oliveira L, Timoteo MA, Correia-de-Sa P. Modulation by adenosine of both muscarinic M1-facilitatory and $\mathrm{M} 2$-inhibition of $[3 \mathrm{H}]$-acetylcholine release from the rat motor nerve terminals. Eur J Neurosci. 2002; 15: 1728-36.

7. Pereira MW, Bornia ECS, Correia-de-Sa P, Alves-Do-Prado W. Presynaptic muscarinic and adenosine receptors are involved in $2 \mathrm{~Hz}$-induced train-of-four fade caused by antinicotinic neuromuscular relaxants in the rat. Clin Exp Pharmacol Physiol 2011; 38: 764-70.

8. Bornia ECS, Correia-de-Sa P, Alves-Do-Prado W. Presynaptic facilitatory adenosine A2A receptors mediate fade induced by neuromuscular relaxants that exhibit anticholinesterase activity. Clin Exp 
Pharmacol Physiol. 2011; 38: 164-9.

9. Tomàs J, Santafè MM, Garcia N, Lanuza MA, Tomàs M, Besalduch N, et al. Presynaptic Membrane Receptors in Acetylcholine Release Modulation in the Neuromuscular Synapse. J Neurosci Res 2014; 92: 543-554.

10. Beny K, Piriou V, Dussart C, Hénaine R, Aulagner G, Armoiry X. Impact of sugammadex on neuromuscular blocking agents use: A multicentric pharmaco-epidemiologic study in French university hospital and military hospitals. Ann Fr Anesth Reanim 2013; 32: 838-43.

11. Pavoni V, Gianesello L, De Scisciolo G, Provvedi E, Horton D, Barbagli R, et al. Reversal of profound and "deep" residual rocuronium-induced neuromuscular blockade by sugammadex: a neurophysiological study. Minerva Anesthesiol 2012; 78: 542-9.

12. Woo T, Kim KS, Shim YH, Kim MK, Yoon SM, Lim YJ, et al. Sugammadex versus neostigmine reversal of moderate rocuronium-induced neuromuscular blockade in Korean patients. Korean $\mathrm{J}$ Anesthesiol 2013; 65: 501-7.

13. Fuchs-Buder T, Meistelman C, Raft J. Sugammadex: clinical development and practical use. Korean J Anesthesiol 2013; 65: 495-500.

14. Kim YB, Lee S, Choi HR, In J, Chang YJ, Kim HJ, et al. Effects of adenosine receptor agonist on the rocuroniuminduced neuromuscular block and sugammadex-induced recovery. Korean J Anesthesiol 2018; 71: 476-82.

15. Haerter F, Simons JC, Foerster U, et al. Comparative effectiveness of calabadion and sugammadex to reverse nondepolarizing neuromuscularblocking agents. Anesthesiology 2015; 123:1337-1349.

16. Garcia N, Priego M, Obis T, Santafe MM, Tomàs M, Besalduch N, et al. Adenosine A1 and A2A receptor-mediated modulation of acetylcholine release in the mice neuromuscular junction. Eur $\mathrm{J}$ Neurosci 2013; 38: 2229-41.

17. Stone TW, Hollins C, Lloyd H. Methylxanthines modulate adenosine release from slices of cerebral cortex. Brain Res 1981; 31: 352-4.

18. Fredholm BB, ljzerman AP, Jacobson KA, Linden J, Müller CE. International union of basic and clinical pharmacology. LXXXI. Nomenclature and classification of adenosine receptors-An update. Pharmacol Rev 2011; 63: 1-34.

19. Bock M, Klippel K, Nitsche B, Bach A, Martin E, Motsch J. Rocuronium potency and recovery characteristics during steady-state desflurane, sevoflurane, isoflurane or propofol anaesthesia. Br J Anaesth 2000; 84: 43-7.

20. Xue FS, Liao X, Tong SY, Liu JH, An G, Luo LK. Dose-response and time-course of the effect of rocuronium bromide during sevoflurane anaesthesia. Anaesthesia 1998; 53: 25-30.

21. Lowry DW, Mirakhur RK, McCarthy GJ, Carroll MT, McCourt KC. Neuromuscular Effects of Rocuronium During Sevoflurane, Isoflurane, and Intravenous Anesthesia. Anesth Analg 1998; 87: 936-40.

22. Kharasch ED, Karol MD, Lanni C, Sawchuk R. Clinical sevoflurane metabolism and disposition. I. Sevoflurane and metabolite pharmacokinetics. Anesthesiology 1995; 82: 1369-78. 


\section{Figures}

Preparation and group allocation

- 40 SD rats enrolled.

- Rat anesthetized with $0.5 \mathrm{mg} / \mathrm{kg}$ Urethane

- Thoracic cage harvested.

- Phrenic nerve-hemidiaphragm tissue obtained

- Immersed in the organ bath filled with oxygenated Krebs buffer

- Phrenic nerve indirect stimulation; TOF with $20 \mathrm{~s}$ interval

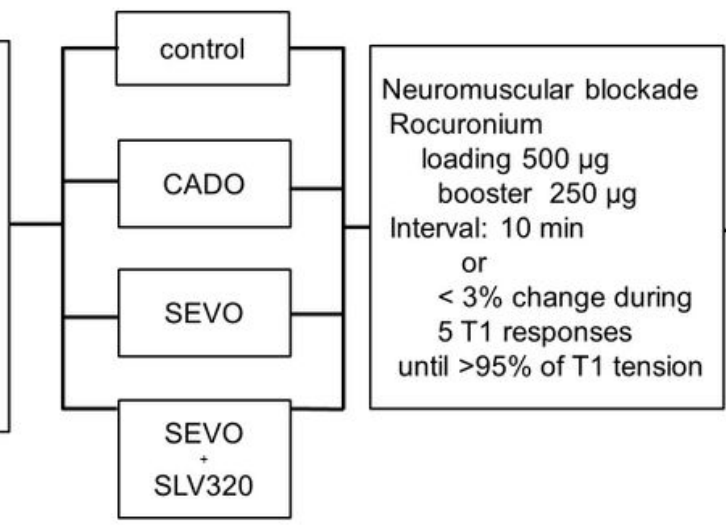

Data collection

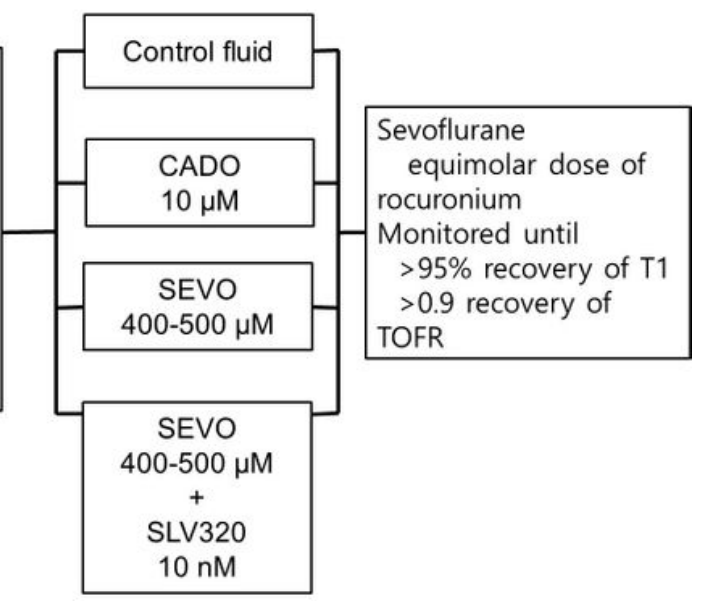

\section{Figure 1}

Study protocol. 10 min of reaction time was allowed after study drugs were added. Abbreviations: CADO, 2-chloroadenosine; SEVO, sevoflurane; SD, Sprague-Dawley rats; TOF, train-of four stimulation. 

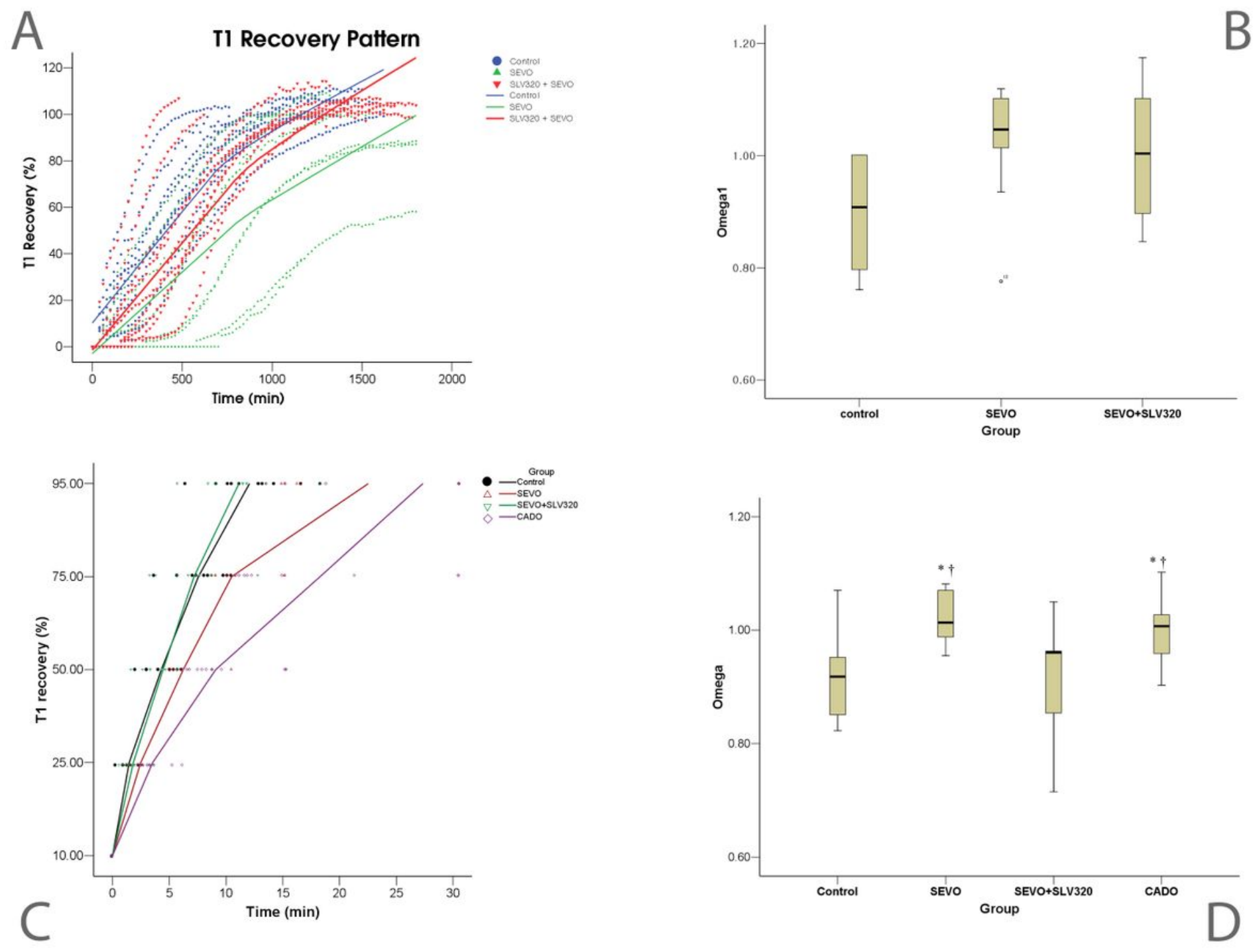

Figure 2

Scatter gram and regression of T1 twitch tension recovery. A) The zero point was set as the time of sugammadex administration. C) The zero point was set as the time when the recovery of the T1 twitch tension was $10 \%$. Comparisons of omegas of $\mathrm{T} 1$ recovery are shown at $\mathrm{B}$ ) and $\mathrm{D}$ ). We set the equation for T1 recovery as $y=50+50 \sin (\Omega x+b)$, where yis the \% recovery of T1 twitch tension and $x$ is time. * $P<$ 0.050 compared with the control. $+\mathrm{P}<0.050$ compared with SEVO + SLV320. Abbreviations: CADO, 2chloroadenosine; SEVO, sevoflurane; TOF, train-of-four stimulation; T1, the first twitch tension of TOF; TOFR, TOF ratio. 


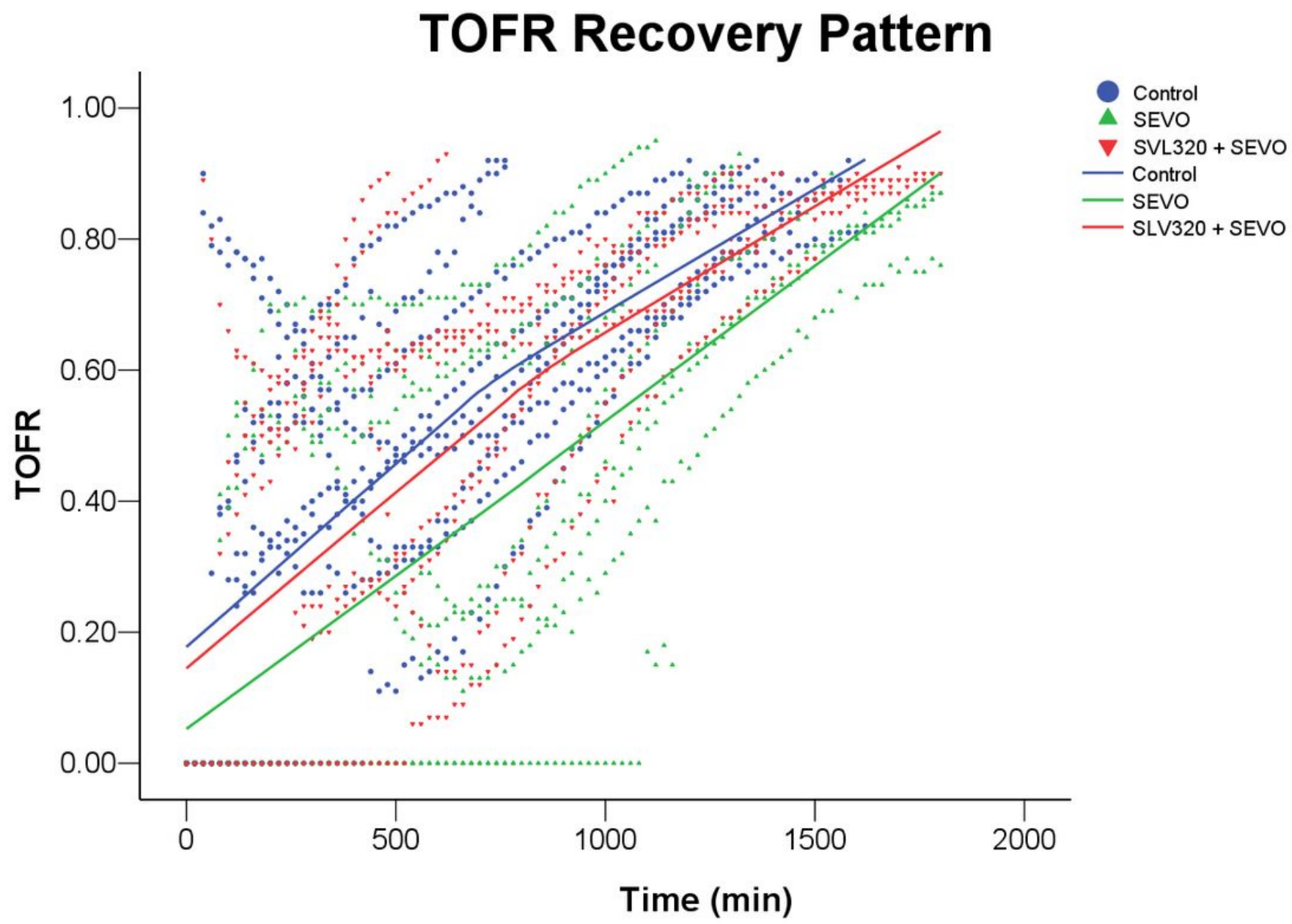

Figure 3

Comparison of TOFR recovery. There were no significant differences among the groups. Data of the CADO group are not shown because the TOFRs of this group did not reach 0.9 within 30 min.

Abbreviations: SEVO, sevoflurane; TOF, train-of-four stimulation; T1, the first twitch tension of TOF; TOFR, TOF ratio.

\section{Supplementary Files}

This is a list of supplementary files associated with this preprint. Click to download.

- ARRIVEguidelines.docx 